\title{
Cortical Electrophysiological Markers of Language Abilities in Children with Hearing Aids: A Pilot Study
}

\author{
David Bakhos, ${ }^{1,2,3}$ Hélène Delage,${ }^{4}$ John Galvin,${ }^{5}$ Emmanuel Lescanne, ${ }^{1,2,3}$ \\ Sylvie Roux,$^{2,3}$ Frédérique Bonnet-Brilhault, ${ }^{2,3,6}$ and Nicole Bruneau ${ }^{2,3}$ \\ ${ }^{1}$ CHRU de Tours, Service ORL et Chirurgie Cervico-Faciale, boulevard Tonnellé, 37044 Tours, France \\ ${ }^{2}$ INSERM U930, 37044 Tours, France \\ ${ }^{3}$ Université François-Rabelais de Tours, CHRU de Tours, UMR-S930, 37044 Tours, France \\ ${ }^{4}$ Laboratoire de Psycholinguistique Expérimentale, Faculté de Psychologie et des Sciences de l'Education, \\ Université de Genève, 40 boulevard du pont d'Arve, 1211 Genève 4, Switzerland \\ ${ }^{5}$ Department of Head and Neck Surgery, David Geffen School of Medicine, UCLA, Los Angeles, CA 90095, USA \\ ${ }^{6}$ CHRU de Tours, Service de Pédopsychiatrie, boulevard Tonnellé, 37044 Tours, France
}

Correspondence should be addressed to David Bakhos; david.bakhos@univ-tours.fr

Received 28 February 2014; Revised 30 June 2014; Accepted 28 July 2014; Published 19 August 2014

Academic Editor: Ramesh Rajan

Copyright (C) 2014 David Bakhos et al. This is an open access article distributed under the Creative Commons Attribution License, which permits unrestricted use, distribution, and reproduction in any medium, provided the original work is properly cited.

\begin{abstract}
Objective. To investigate cortical auditory evoked potentials (CAEPs) in pediatric hearing aid (HA) users, with and without language impairment. Design. CAEPs were measured in 11 pediatric HA users (age: 8-12 years) with moderate bilateral sensorineural hearing loss (HL); participants were classified according to language ability. CAEPs were also measured for a control group of 11 age-matched, normal-hearing $(\mathrm{NH})$ children. Results. HL children without language impairment exhibited normal CAEPs. HL children with language impairment exhibited atypical temporal CAEPs, characterized by the absence of N1c; frontocentral responses displayed normal age-related patterns. Conclusion. Results suggest that abnormal temporal brain function may underlie language impairment in pediatric HA users with moderate sensorineural HL.
\end{abstract}

\section{Introduction}

The human cochlea is mature at birth; however, axonal, dendritic, and synaptic maturation and myelination continue to develop in the brainstem into early childhood and in the cerebral cortex into late childhood [1]. Auditory development and speech perception are guided by relevant acoustic and linguistic information experienced early in life to assure cortical maturation [2]. Hearing loss (HL) can be deleterious to children's speech and language development due to reduced quality and quantity of auditory input, and these developmental difficulties can have a cascading effect on social, academic, and (later) occupational success [3]. Given the restricted auditory input, abnormal cortical auditory maturation can occur in children with sensorineural HL, suggesting that the root cause may lie in the inner ear [4].

In case of moderate HL in childhood, hearing aids (HAs) can improve speech audibility and facilitate language development, assuming that the auditory cortical areas are functional [5]. However, individual variation in language performance has been observed in children with mild to moderate sensorineural HL [6]. Approximately 50\% of children fitted with HAs for moderate HL have language impairment despite normal aided audiometric thresholds $[7,8]$; the same finding was observed in adolescents [9]. Other studies have suggested that impairment in basic auditory processing might contribute to language difficulties in children with specific language impairment [10-12]. Language impairment might therefore be related to abnormal cortical auditory processing, which can be investigated using cortical auditory evoked potentials (CAEPs).

The successive peaks of CAEPs correspond to the spatiotemporal involvement of the cortical auditory generators and are therefore influenced by cortical maturation [13-15]. Whereas the morphology of frontocentral CAEPs is strongly influenced by age and provides an important 
index of auditory function and plasticity, the morphology of temporal CAEPs remains stable throughout childhood [16], with the successive negative N1a and N1c peaks occurring at approximately 80 and $160 \mathrm{~ms}$, respectively [17]. These temporal responses (T-complex) represent the activity of the secondary auditory cortex [17]. The frontocentral CAEPs of children mainly exhibit two successive positive-negative peaks (P100 and N250) occurring at approximately 100 and $200 \mathrm{~ms}$, respectively. At 8-11 years, adult-like CAEP waveforms progressively emerge, with the successive N1b, $\mathrm{P} 2$, and N250 peaks occurring at approximately 100, 180, and $220 \mathrm{~ms}$, respectively. Given that speech information is transmitted at somewhat rapid rates (100 to 200 words per minute) [18], short interstimulus intervals $(<750 \mathrm{~ms})$ have sometimes been used to study the effect of the stimulus rate on frontocentral CAEPs in children that were categorized according to age [13]. Indeed, stimulus rate has been recognized as a marker of cortical auditory maturation [13]. Only one study has examined the influence of stimulus rate on temporal responses in children [19]. In that study, the amplitude of the temporal negative peak corresponding to Tb increased with interstimulus interval (350, 700, 1400, and $2000 \mathrm{~ms}$ ), indicating a long refractory period for the underlying generator. Thus, it appears that long interstimulus intervals may be favorable for identifying successive peaks of the T-complex, and their asymmetry.

The aim of this study was to use CAEPs to investigate cortical auditory processing in regard to pediatric HA users with different levels of language ability. We hypothesized that temporal auditory responses and/or their sensitivity to stimulus rate would reflect different levels of language ability in these patients.

\section{Patients and Method}

2.1. Subjects. In this cortical electrophysiological study, we included children with symmetrical bilateral sensorineural HL fitted with HAs and aged between 8 and 12 years. Children in this age range were chosen for this pilot study as they are likely to understand and follow instructions during testing (i.e., CAEP, audiometry, and language tests). Participants were recruited from the Pediatric Unit of the Otolaryngology Department during clinical follow-up visits.

We reviewed patient charts of pediatric HA users. Thirty children had symmetrical bilateral sensorineural HL and were between 8 and 12 years old. Eleven children ( 8 males, 3 females), aged between 8.3 and 12.8 years (mean: 10.9 yrs), fitted with bilateral HAs for a bilateral moderate sensorineural HL were accepted into the study. A control group of 11 age- and gender-matched $\mathrm{NH}$ children with normal language development, as evaluated using the battery of oral language (evaluated with BILO battery including receptive and expressive language skills; see below for explanation), was also recruited for the study. For all participants, French was the main language spoken at home. All participants were right-handed.

Aided and unaided pure tone average (PTA) thresholds (averaged across audiometric frequencies $0.5,1,2$, and $4 \mathrm{kHz}$ ) were $<20 \mathrm{~dB}$ for pediatric HA and NH participants, respectively. Demographic data for pediatric HA users are shown in Table 1. All participants used oral communication and were enrolled in mainstream schools.

The Ethics Committee of the University Hospital of Tours approved the protocol, and written informed consent was obtained from the parents and assent from the children.

Spoken language and literacy skills were assessed using BILO, a set of standardized, computerized French language tests [20]. The BILO battery was standardized over a population of 272 primary and middle school students. In the BILO battery, phonology is assessed by a word repetition task using 42 words of increasing length and/or complexity. Expressive vocabulary is assessed using a classical naming task. Expressive grammar is evaluated using a sentence completion task and assesses the ability to produce a variety of specific grammatical morphemes: nominal, adjectival, and verbal inflexion, irregular plurals, prepositions, passive structure, and pronominal clitics. Reading is assessed using a timed task in which the child has to read, in 60 seconds, as many words as possible from a list of words of increasing difficulty. Spelling is tested using a word identification task, in which the child is presented with words that are correctly spelled, contain homophonic or nonhomophonic misspellings, or merely belong to the same semantic field and must decide whether the word corresponding to the picture is correct. Lexical judgment is tested by asking the child to decide whether there is concordance between a word and picture presented simultaneously. Grammatical judgment is tested by asking the child to decide whether a sentence corresponding to a picture is grammatically correct.

As described in Delage and Tuller [9], BILO scores were converted to $\mathrm{z}$-scores. Language impairment was defined as scores on two or more of the language subtests that were 1.2 standard deviations below the norm. Six of the pediatric HA participants were deemed as having "good" language ability $(\mathrm{HL}+)$, with BILO > 1.2, while five were deemed as having "fair" language ability ( $\mathrm{HL}-$ ), with BILO $<1.2$ (see Table 1). They were age-matched to control group of $11 \mathrm{NH}$ children categorized according to similar language ability (HL+controls and HL- controls). Six children (4 males and 2 females) were included in the HL+ controls (mean age: 11.6 years \pm 0.7 ) and 5 children ( 4 males and one female) were included in the $\mathrm{HL}-$ controls (mean age: 9.4 years \pm 1.9 ).

\subsection{CAEPs Assessments}

2.2.1. Stimuli and Procedure. Participants were tested while sitting on an armchair in a dimly lit, sound-insulated room; pediatric HA participants were tested while wearing their HAs. Participants' mother or father accompanied them in the room during testing. The stimuli were comprised of 50ms tone bursts $(1100 \mathrm{~Hz})$ delivered through two loudspeakers placed symmetrically on each side of the computer screen. The tone stimuli were presented via Neuroscan Stim ${ }^{2}$ software. The stimuli were presented at four different interstimulus intervals: 700 (i1), 1100 (i2), 1500 (i3), and 3000 (i4) ms. 
TABLE 1: Demographic information for the pediatric HA users with bilateral moderate sensorineural HL.

\begin{tabular}{|c|c|c|c|c|c|c|c|c|c|c|}
\hline Subject & Gender & $\begin{array}{c}\text { Age } \\
\text { test } \\
\text { (yrs; months) }\end{array}$ & $\begin{array}{l}\text { Duration of } \\
\text { auditory } \\
\text { deprivation } \\
\text { (months) }\end{array}$ & $\begin{array}{c}\text { Age at which } \\
\text { child received } \\
\text { HA } \\
\text { (yrs; months) }\end{array}$ & $\begin{array}{c}\text { Experience xith } \\
\text { HA } \\
\text { (yrs; months) }\end{array}$ & Aetiology & $\begin{array}{l}\text { Right } \\
\text { PTA }\end{array}$ & $\begin{array}{l}\text { Left } \\
\text { PTA }\end{array}$ & $\begin{array}{l}\text { BILO } \\
\text { score }\end{array}$ & Group \\
\hline 1 & M & $8 ; 3$ & 6 & $3 ; 5$ & $4 ; 10$ & Unknown & 63 & 69 & -1.34 & $\mathrm{HL}-$ \\
\hline 2 & M & $8 ; 5$ & 3 & $2 ; 1$ & $6 ; 4$ & Familial & 64 & 65 & -3.53 & $\mathrm{HL}-$ \\
\hline 3 & M & $8 ; 11$ & 4 & 2 & $6 ; 11$ & Familial & 66 & 66 & -1.46 & HL- \\
\hline 4 & $\mathrm{~F}$ & $9 ; 7$ & 2 & $6 ; 1$ & $3 ; 6$ & Familial & 56 & 56 & -5.31 & HL- \\
\hline 5 & M & $10 ; 5$ & 2 & $5 ; 11$ & $4 ; 6$ & Unknown & 45 & 41 & -0.97 & $\mathrm{HL}+$ \\
\hline 6 & M & $11 ; 5$ & 2 & $5 ; 11$ & $5 ; 6$ & Unknown & 41 & 43 & -0.2 & $\mathrm{HL}+$ \\
\hline 7 & M & $11 ; 8$ & 1 & $3 ; 5$ & $8 ; 3$ & Familial & 55 & 66 & -0.46 & $\mathrm{HL}+$ \\
\hline 8 & $\mathrm{~F}$ & $12 ; 1$ & 18 & $8 ; 11$ & $3 ; 2$ & Unknown & 40 & 41 & -0.16 & $\mathrm{HL}+$ \\
\hline 9 & $\mathrm{~F}$ & $12 ; 2$ & 6 & $4 ; 10$ & $7 ; 4$ & Familial & 59 & 45 & 1.31 & $\mathrm{HL}+$ \\
\hline 10 & M & $12 ; 6$ & 5 & $8 ; 5$ & $4 ; 1$ & Familial & 41 & 41 & -1.25 & HL- \\
\hline 11 & M & $12 ; 10$ & 6 & $5 ; 2$ & $7 ; 8$ & Unknown & 41 & 42 & -0.5 & HL+ \\
\hline
\end{tabular}

Note: M: male; F: female; yrs: years; HA: hearing aid; PTA: aided pure tone audiometric threshold averaged over 0.5, 1, 2, and 4 kHz; BILO: Batterie Informatisée du Langage Oral; HL+: good language ability; HL-: fair language ability.

The sound intensity was $70 \mathrm{dBA}$ measured at the head of the participant.

2.2.2. Electroencephalogram (EEG) Recording. EEG recordings were obtained using $28 \mathrm{Ag}-\mathrm{AgCl}$ cup electrodes ( $\mathrm{Ffz}, \mathrm{Fz}$, $\mathrm{Cz}, \mathrm{Pz}, \mathrm{O} 1, \mathrm{~F} 3, \mathrm{FCl}, \mathrm{FT} 3, \mathrm{C} 3, \mathrm{~T} 3, \mathrm{CP} 1, \mathrm{TP} 3, \mathrm{P} 3, \mathrm{~T} 5$, and F7) and their counterparts on the right hemiscalp. Electrodes were placed according to the 10-20 system, as well as the left and right mastoids (M1 and M2), and referenced to the nose. In addition, to detect ocular artifacts, vertical electrooculogram (EOG) data were recorded from two electrodes above and below the right eye (vertical bipolar).

The EEG and EOG were digitized (Neuroscan Synamps amplifier, Scan 4.3, Compumedics Corp., El Paso, TX) at a sampling rate of $500 \mathrm{~Hz}$. The EEG was amplified and bandpass-filtered $(0.3-100 \mathrm{~Hz})$. Electrode impedances were kept below $10 \mathrm{k} \Omega$. Eye movement artifacts were eliminated using a spatial filter transform developed by Neuroscan, and EEG periods with movement artifacts were rejected manually. A digital zero-phase-shift low-pass filter $(30 \mathrm{~Hz})$ was then applied to the EEG.

2.2.3. Data Analysis. CAEPs were analyzed with the ELAN software [21]. Analysis was performed for waveform peaks occurring at frontocentral sites (N1b-P2-N250) and at temporal sites: Nla, Nlc, Plt (the positive peak between these Nla and N1c), and P2t (the positive peak following N1c).

The influence of interstimulus interval on each peak of the CAEPs was analyzed using Friedman nonparametric analyses of variance. Amplitudes and latency peaks measured in HL+ and HL- children and the control group were compared using nonparametric Mann-Whitney rank tests.

\section{Results}

HL+ and HL- children did not differ, using Wilcoxon MannWhitney test, on age at testing (HL+: 11.7 years old \pm 0.8 ; HL-: 9.5 years old $\pm 1.7 ; P=0.08$ ) or experience with hearing aids (HL+: 6.4 years, $\mathrm{HL}-: 5.7$ years; $P=0.4$ ).

3.1. Frontocentral Responses. Frontocentral CAEPs displayed similar successive N1b-P2-N250 peaks across the four groups. No significant difference was observed for the N1b peak amplitudes and latencies between HL+ and HL+ controls, or between HL- and HL- controls (Figure 1). The N1b peak amplitude was smaller for HL- than for HL+ participants and for HL- controls than for HL+ controls. This might be related to age differences, as the HL- and HL- control participants were younger (mean age was, resp., 9.5 and 9.4 years) than the HL+ and HL+ control participants (mean age was, resp., 11.7 and 11.6 years). A significant effect of interstimulus interval was observed on N1b amplitude for all groups except for the HL-. The N1b peak increased with interstimulus interval in $\mathrm{HL}+(P=0.01), \mathrm{HL}+$ controls $(P=0.03)$, and HL- controls $(P=0.04)$. There was a greater $\mathrm{P} 2$ peak amplitude in HL+ than in HL+ controls and was significant at i3 $(P=0.02)$ and at i4 $(P=0.004)$. The P2 peak was better individualized in $\mathrm{HL}$ - children than in matched controls. The N250 peak amplitude and latency did not vary with interstimulus interval and no significant differences across groups were observed.

3.2. Temporal Responses. Because no significant effect of interstimulus interval was found for the amplitude and latency of the successive peaks recorded at temporal sites Nla, P1t, N1c, and P2t, the CAEPs were averaged across the 4 interstimulus intervals to increase the signal to noise ratio (SNR). 


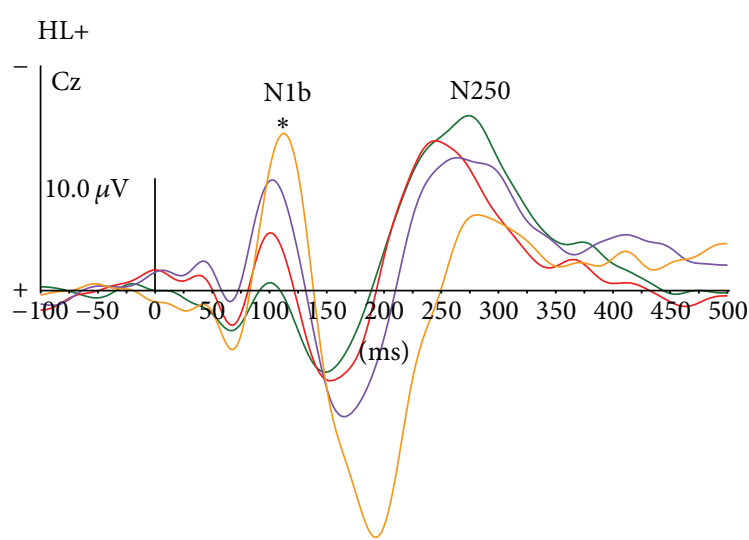

P2

(a)

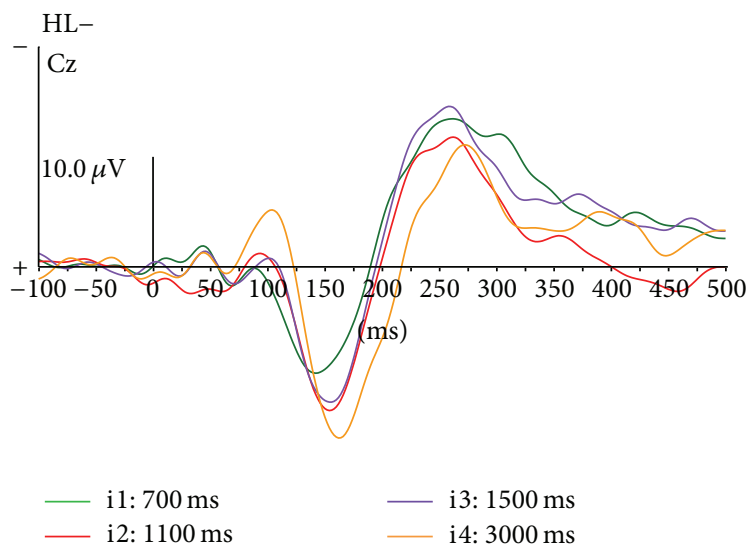

(c)

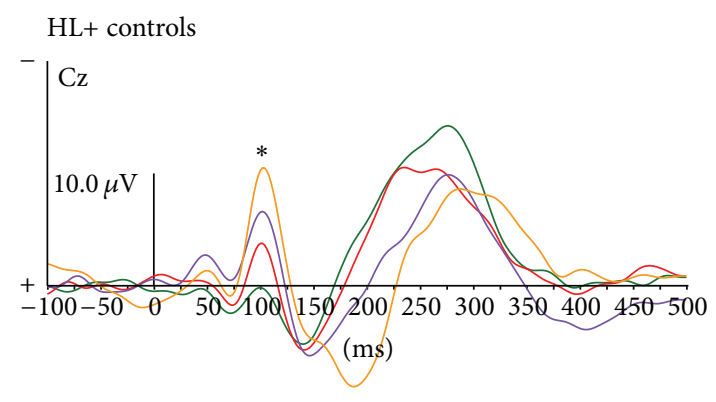

(b)

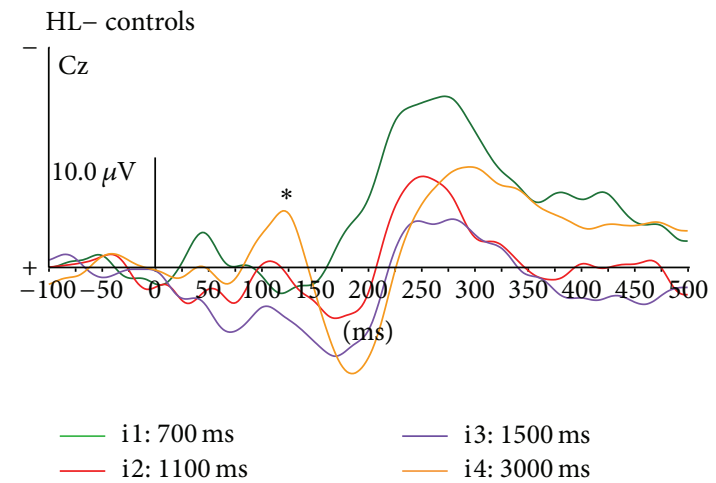

(d)

Figure 1: Cortical AEPs. Midline responses (Cz) at different interstimulus intervals (i1, i2, i3, and i4) for the HL+ (a), HL+ controls (b), HL(c), and HL- controls (d). The asterisks indicate significant effect of interstimulus interval $(P<0.05)$.

Although the grand average Nla peak amplitude was greater in the HL+ and HL- groups than in their respective controls (mainly on the left temporal site), the difference was not statistically significant. The subsequent P1t, N1c, and P2t waves also were not significantly different between the HL+ and HL+ control groups. However, the N1c and P2t amplitudes were significantly smaller (only on the right temporal site) in the HL- group than in the $\mathrm{HL}-$ controls (N1c: HL $-=-0.8 \mu \mathrm{V} \pm 1.3$, HL - controls $=-3.1 \mu \mathrm{V} \pm 0.9, \mathrm{U}=$ 2, $P=0.03$; P2t: HL $-=3.1 \mu \mathrm{V} \pm 0.6, \mathrm{HL}+$ controls $=5.4 \mu \mathrm{V} \pm$ $1.4, \mathrm{U}=2, P=0.03$ ). This difference between the HL- group and the HL-controls was not observed for either latency or amplitude of the preceding P1t (Figure 2).

\section{Discussion}

The present pilot study provided interesting preliminary findings regarding the relationship between CAEP characteristics and language ability in 8- to 12 -year-old pediatric HA users. Atypical CAEPs were observed at temporal recording sites for pediatric HA users with some degree of language impairment. In normal development, temporal CAEPs typically display a stable morphology through childhood and particularly for the age range of the present study, with successive Nla and Nlc peaks [16, 17], suggesting that cortical auditory maturation does not change the morphology of temporal responses for children between 8 and 12 years old.

In our study, despite a normal (or greater than normal) amplitude of the early temporal peaks (Nla, P1t) for all pediatric HA patients, the later temporal responses (N1c and P2t) were reduced or absent in the HL- group. This does not appear to be due to an absence of cortical auditory input because waves Nla and P1t were present and normal in all the pediatric HA patients. Nlc wave was absent or smaller in the HL- group. This relationship between N1c abnormalities (N1c being reduced or absent) and language impairment has previously been shown in other clinical populations with language impairment such as in children with autism [22], Down's syndrome [23], or specific language impairment [24]. Because generators of Nlc are located at the lateral part of the superior temporal gyrus [25-27], the present results emphasize the importance of these cortical areas in language processing.

Relatively few studies have investigated CAEP temporal responses in pediatric HA users. Most of these studies have focused on frontocentral responses (especially the latency of 

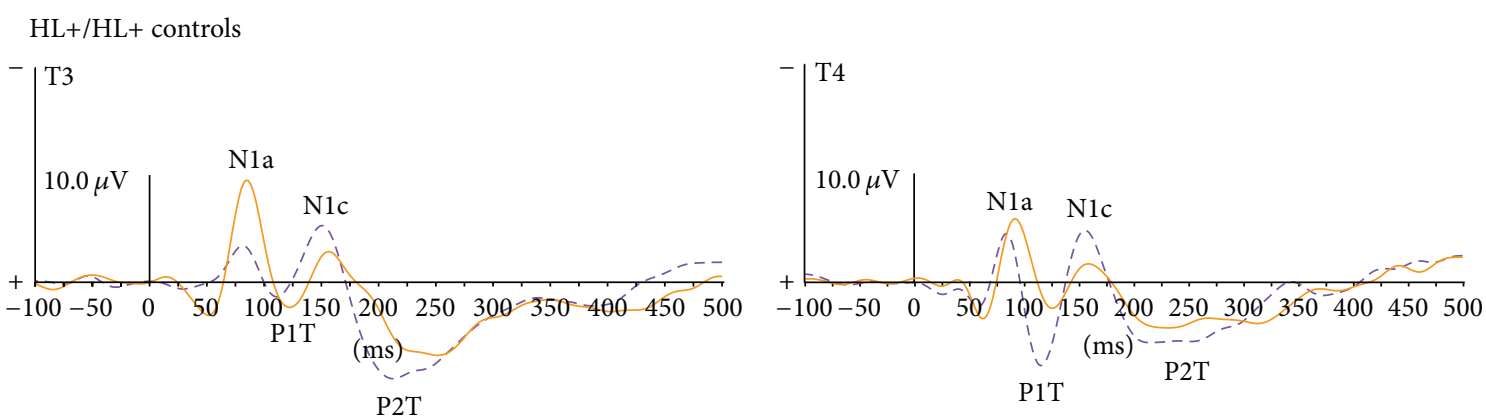

$\begin{array}{ll}- & \mathrm{HL}+ \\ ---\mathrm{HL}+\text { controls }\end{array}$

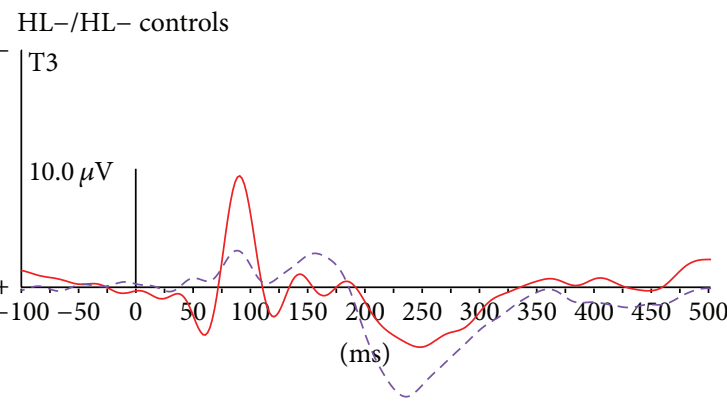

- HL-

- - - HL- controls
- HL+

_. - HL+ controls

(b)

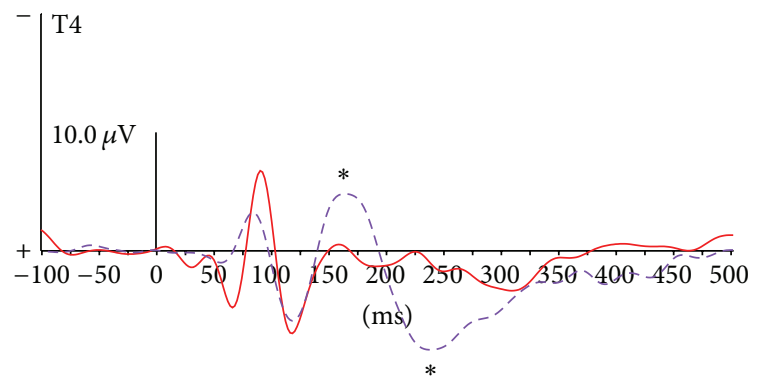

$-\mathrm{HL}-$
-- HL- controls

(c)

(d)

Figure 2: CAEP T3 (a and c) and T4 temporal responses (b and d) at i1, i2, i3, and i4 for the HL+ and HL+ control groups (a and b) and for the HL- and HL- control groups ( $c$ and d). The asterisks indicate significant differences $(P<0.05)$ for the peak amplitude wave.

the P1 peak recorded at the vertex) in deaf children who use cochlear implants [28-30]. These studies found that P1 peak latency can be a biomarker of auditory cortex maturation in children with congenital hearing loss and that this P1 peak latency will decrease with auditory rehabilitation. In the present study, the P1 peak latency did not differ between HA patients and $\mathrm{NH}$ controls, suggesting that the HA provided enough auditory input to allow maturation of the primary auditory cortex as found in cochlear-implanted children.

Unlike the temporal responses, the frontocentral responses of normally developing children are greatly influenced by age. The smaller N1b peak amplitude in the HL- and HL- control groups, compared to the well-defined peaks and greater amplitude of N1b in the HL+ and HL+ control groups, might be due to age differences between groups. This result is in agreement with the literature indicating the emergence of $\mathrm{Nlb}$ at approximately $8-10$ years of age and greater N1b amplitude at 10-12 years of age. The greater $\mathrm{P} 2$ peak amplitude observed in HL+ and HL- groups than in the controls may be related to HA amplification, as described in previous studies [31,32]. N1b peak amplitude increased with interstimulus interval in the HL+ group, similar to the HL+ controls and previous studies [5]; the effect of interstimulus interval was not observed for the HLgroup. This finding might be related to language impairment or the younger age of the HL- group, as a significant effect of interstimulus interval was observed for the HL- controls.

In this study, children with HA and good language ability $(\mathrm{HL}+)$ were older than those with language impairment (HL-). Language abilities were evaluated with BILO, which is calibrated in order to allow comparisons in language ability across age groups. Moreover, CAEPs were compared with age- and gender-matched control children.

Although further longitudinal studies are needed with larger sample of children, these preliminary results suggest that abnormal CAEP responses recorded at temporal sites might underlie language impairment in pediatric HA users who have a moderate sensorineural HL.

\section{Conflict of Interests}

The authors declare that there is no conflict of interests regarding the publication of this paper.

\section{Acknowledgment}

This study was supported by the French ENT Society. 


\section{References}

[1] J. K. Moore and F. H. Linthicum Jr., "The human auditory system: a timeline of development," International Journal of Audiology, vol. 46, pp. 460-478, 2007.

[2] P. K. Kuhl, "A new view of language acquisition," Proceedings of the National Academy of Sciences of the United States of America, vol. 97, no. 22, pp. 11850-11857, 2000.

[3] H. W. Catts, M. E. Fey, J. B. Tomblin, and X. Zhang, "A longitudinal investigation of reading outcomes in children with language impairments," Journal of Speech, Language, and Hearing Research, vol. 45, no. 6, pp. 1142-1157, 2002.

[4] J. K. Moore, J. K. Niparko, M. R. Miller, and F. H. Linthicum, "Effect of profound hearing loss on a central auditory nucleus," The American Journal of Otology, vol. 15, no. 5, pp. 588-595, 1994.

[5] J. B. Tombin, J. J. Oleson, S. E. Ambrose, E. Walker, and M. P. Moeller, "The influence of hearing aids on the speech and language development of children with hearing loss," JAMA Otolaryngology-Head \& Neck Surgery, vol. 140, pp. 403-409, 2014.

[6] J. Briscoe, D. V. Bishop, and C. F. Norbury, "Phonological processing, language, and literacy: a comparison of children with mild-to-moderate sensorineural hearing loss and those with specific language impairment," The Journal of Child Psychology and Psychiatry, vol. 42, no. 3, pp. 329-340, 2001.

[7] K. Hansson, J. Forsberg, A. Löfqvist, E. Mäki-Torkko, and B. Sahlén, "Working memory and novel word learning in children with hearing impairment and children with specific language impairment," International Journal of Language and Communication Disorders, vol. 39, no. 3, pp. 401-422, 2004.

[8] P. G. Stelmachowicz, A. L. Pittman, B. M. Hoover, and D. E. Lewis, "Novel-word learning in children with normal hearing and hearing loss," Ear and Hearing, vol. 25, no. 1, pp. 47-56, 2004.

[9] H. Delage and L. Tuller, "Language development and mildto-moderate hearing loss: does language normalize with age?" Journal of Speech, Language, and Hearing Research, vol. 50, no. 5, pp. 1300-1313, 2007.

[10] P. Tallal, "Rapid auditory processing in normal and disordered language development," Journal of Speech and Hearing Research, vol. 19, no. 3, pp. 561-571, 1976.

[11] B. A. Wright, L. J. Lombardino, W. M. King, C. S. Puranik, C. M. Leonard, and M. M. Merzenich, "Deficits in auditory temporal and spectral resolution in language- impaired children," Nature, vol. 387, no. 6629, pp. 176-178, 1997.

[12] G. M. McArthur and D. V. Bishop, "Which people with specific language impairment have auditory processing deficits?" Cognitive Neuropsychology, vol. 21, pp. 79-94, 2004.

[13] E. Sussman, M. Steinschneider, V. Gumenyuk, J. Grushko, and K. Lawson, "The maturation of human evoked brain potentials to sounds presented at different stimulus rates," Hearing Research, vol. 236, no. 1-2, pp. 61-79, 2008.

[14] C. W. Ponton, J. J. Eggermont, B. Kwong, and M. Don, "Maturation of human central auditory system activity: evidence from multi-channel evoked potentials," Clinical Neurophysiology, vol. 111, no. 2, pp. 220-236, 2000.

[15] J. L. Wunderlich and B. K. Cone-Wesson, "Maturation of CAEP in infants and children: a review," Hearing Research, vol. 212, no. 1-2, pp. 212-223, 2006.

[16] I. Tonnquist-Uhlen, C. W. Ponton, J. J. Eggermont, B. Kwong, and M. Don, "Maturation of human central auditory system activity: the T-complex," Clinical Neurophysiology, vol. 114, no. 4, pp. 685-701, 2003.

[17] N. Bruneau, S. Roux, P. Guërin, C. Barthélémy, and G. Lelord, "Temporal prominence of auditory evoked potentials (N1 wave) in 4-8- year-old children," Psychophysiology, vol. 34, no. 1, pp. 32-38, 1997.

[18] J. C. Krause and L. D. Braida, "Investigating alternative forms of clear speech: the effects of speaking rate and speaking mode on intelligibility," Journal of the Acoustical Society of America, vol. 112, pp. 2165-2172, 2002.

[19] R. Ceponiene, M. Cheour, and R. Näätänen, "Interstimulus interval and auditory event-related potentials in children: evidence for multiple generators," Electroencephalography and Clinical Neurophysiology, vol. 108, no. 4, pp. 345-354, 1998.

[20] A. Khomsi, J. Khomsi, F. Pasquet, and A. Parbeau-Gueno, Bilan Informatisé du Langage Oral (BILO), Editions du Centre de Psychologie Appliquée, Paris, France, 2007.

[21] P.-E. Aguera, K. Jerbi, A. Caclin, and O. Bertrand, "ELAN: a software package for analysis and visualization of MEG, EEG, and LFP signals," Computational Intelligence and Neuroscience, vol. 2011, Article ID 158970, 11 pages, 2011.

[22] N. Bruneau, S. Roux, J. L. Adrien, and C. Barthélémy, "Auditory associative cortex dysfunction in children with autism: evidence from late auditory evoked potentials (N1 wave-T complex)," Clinical Neurophysiology, vol. 110, no. 11, pp. 1927-1934, 1999.

[23] M. A. Groen, P. Alku, and D. V. M. Bishop, "Lateralisation of auditory processing in Down syndrome: a study of T-complex peaks Ta and Tb," Biological Psychology, vol. 79, no. 2, pp. 148$157,2008$.

[24] V. L. Shafer, R. G. Schwartz, and B. Martin, "Evidence of deficient central speech processing in children with specific language impairment: The T-complex," Clinical Neurophysiology, vol. 122, no. 6, pp. 1137-1155, 2011.

[25] G. G. Celesia, "Organization of auditory cortical areas in man," Brain, vol. 99, no. 3, pp. 403-414, 1976.

[26] C. C. Wood and J. R. Wolpaw, "Scalp distribution of human auditory evoked potentials. II. Evidence for overlapping sources and involvement of auditory cortex," Electroencephalography and Clinical Neurophysiology, vol. 54, no. 1, pp. 25-38, 1982.

[27] M. Scherg and D. von Cramon, "Evoked dipole source potentials of the human auditory cortex," Electroencephalography and Clinical Neurophysiology, vol. 65, no. 5, pp. 344-360, 1986.

[28] C. W. Ponton, M. Don, J. J. Eggermont, M. D. Waring, and A. Masuda, "Maturation of human cortical auditory function: differences between normal-hearing children and children with cochlear implants," Ear and Hearing, vol. 17, no. 5, pp. 430-437, 1996.

[29] A. Sharma, M. F. Dorman, and A. J. Spahr, "A sensitive period for the development of the central auditory system in children with cochlear implants: implications for age of implantation," Ear and Hearing, vol. 23, no. 6, pp. 532-539, 2002.

[30] A. Sharma, K. Martin, P. Roland et al., "P1 latency as a biomarker for central auditory development in children with hearing impairment," Journal of the American Academy of Audiology, vol. 16, no. 8, pp. 564-573, 2005. 
[31] P. A. Korczak, D. Kurtzberg, and D. R. Stapells, "Effects of sensorineural hearing loss and personal hearing aids on cortical event-related potential and behavioral measures of speechsound processing," Ear and Hearing, vol. 26, no. 2, pp. 165-185, 2005.

[32] C. J. Billings, K. L. Tremblay, P. E. Souza, and M. A. Binns, "Effects of hearing aid amplification and stimulus intensity on cortical auditory evoked potentials," Audiology and Neurotology, vol. 12, no. 4, pp. 234-246, 2007. 


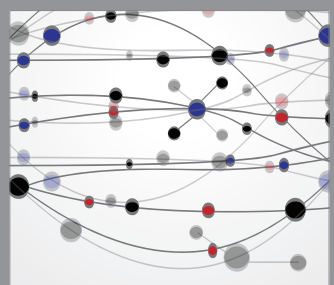

The Scientific World Journal
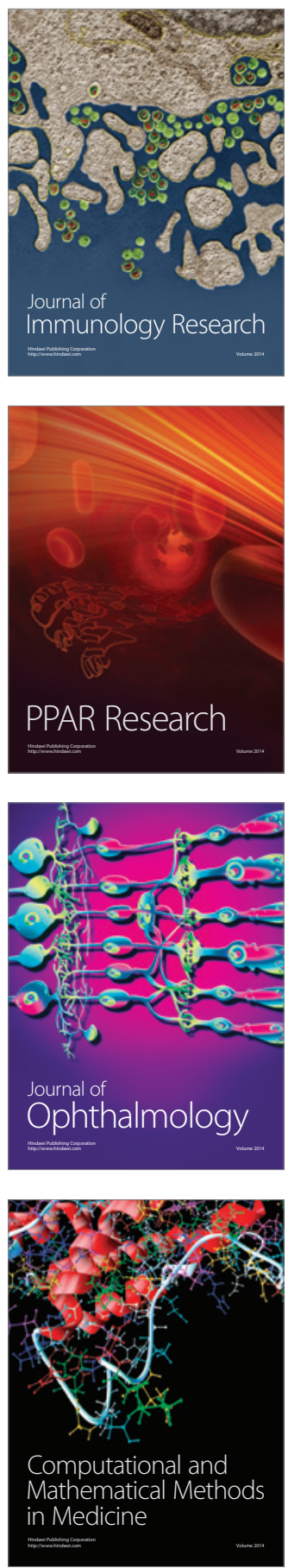

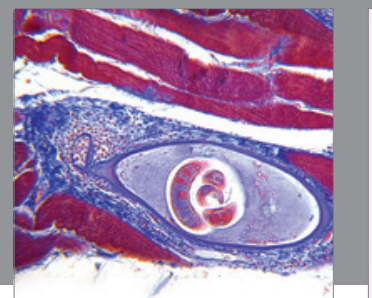

Gastroenterology

Research and Practice
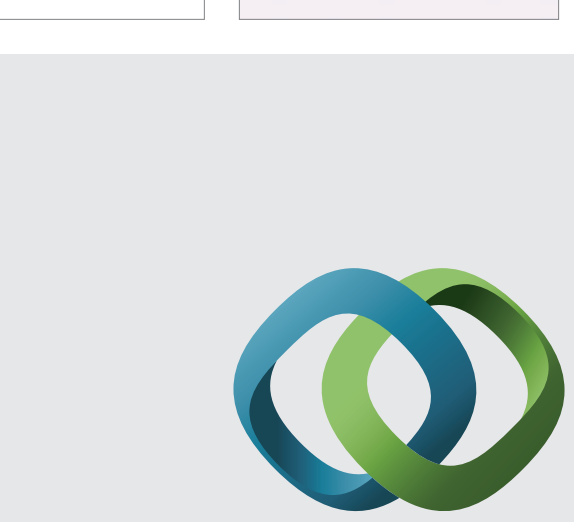

\section{Hindawi}

Submit your manuscripts at

http://www.hindawi.com
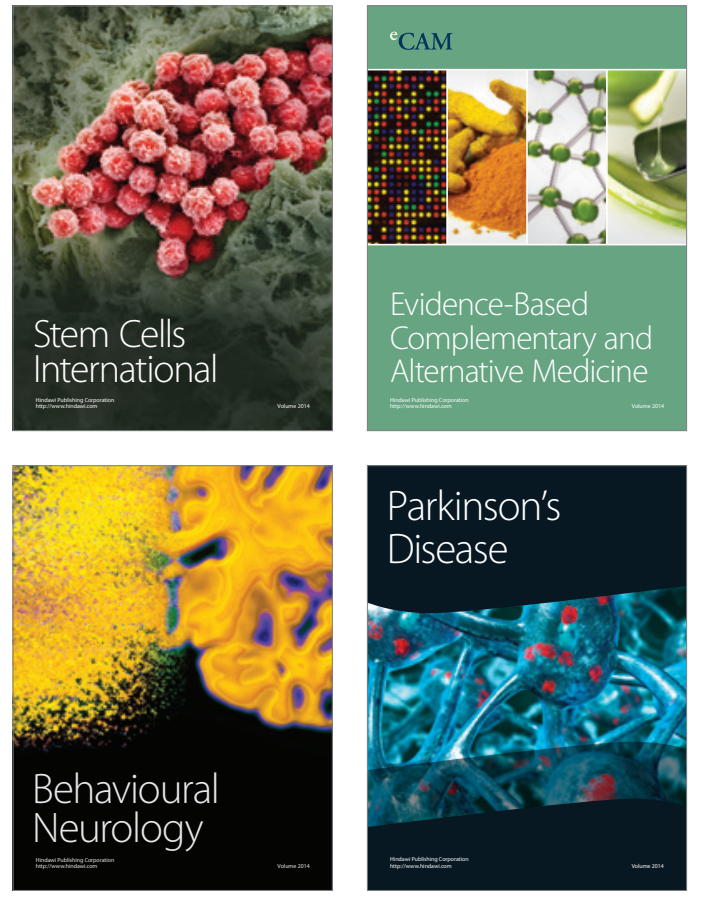
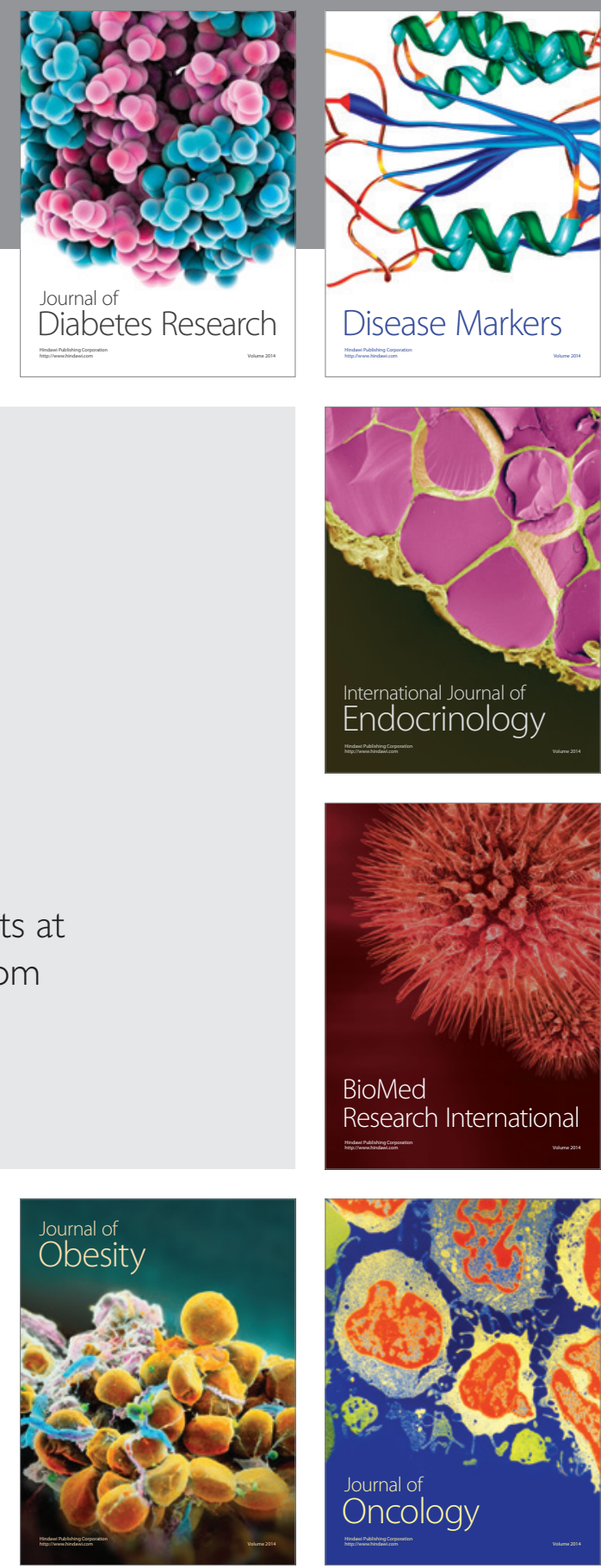

Disease Markers
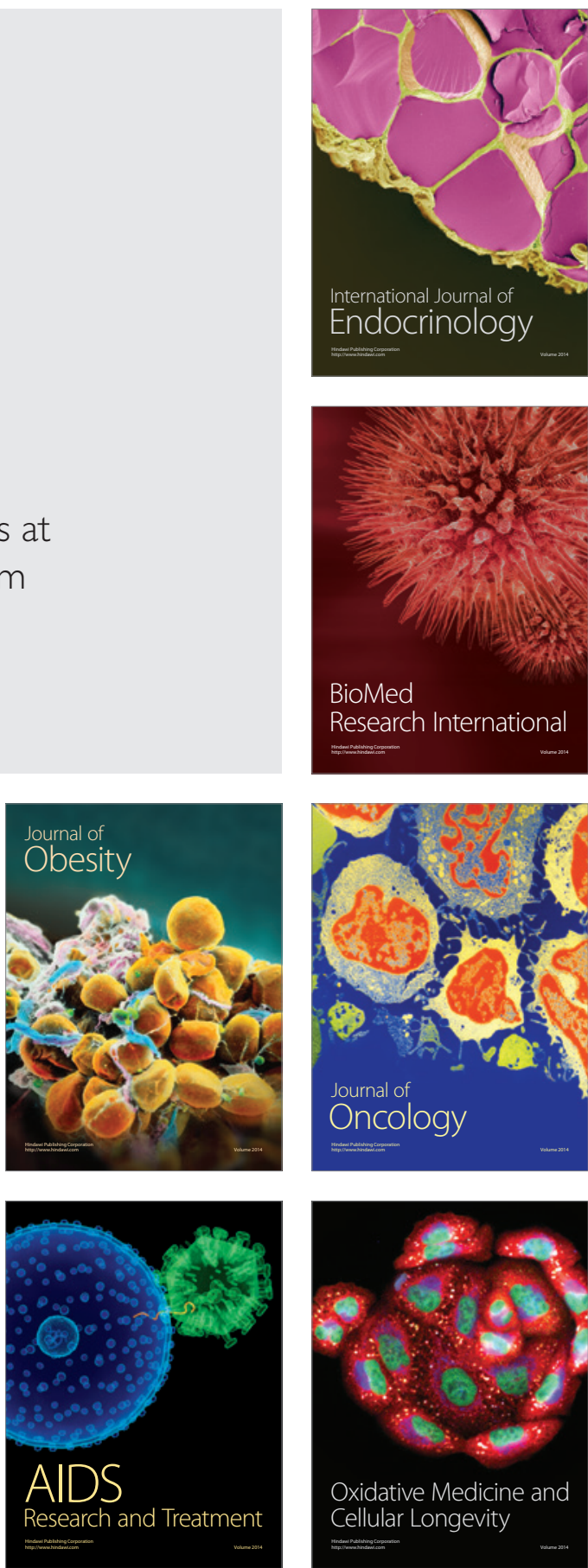